\title{
Análisis de la producción y la comercialización del banano como aspecto socio económico.
}

\section{Analysis of the production and marketing of bananas as socio-economic}

aspect.

Velasteguí López Efraín. ${ }^{1}$, Herrera Vargas Erik. ${ }^{2}$

\section{Resumen.}

DOI: https://doi.org/10.33262/visionariodigital.v1i2.241

Los bananos y plátanos son cultivados perennes que crecen con rapidez y pueden cosecharse durante todo el año en el año 2000 se cultivó una superficie de alrededor de 9 millones de hectáreas. El promedio de la producción mundial en 1998-2000 fue de 92 millones de toneladas anuales y en 2001 se estimó en 99 millones de toneladas. Estas cifras son una aproximación, ya que la mayor parte de la producción mundial de banano, casi el 85 por ciento, procede de parcelas relativamente pequeñas y huertos familiares en donde no hay estadísticas. Ecuador es un país privilegiado y reconocido internacionalmente como el primer exportador bananero en el mundo, destacándose por la calidad de su fruta. Corporación Palmar ocupa un lugar muy importante en el desarrollo económico del Ecuador, dados los altos volúmenes de exportación bananera que realiza a mercados cuyos estándares de calidad son sumamente exigentes.

Tan solo durante la primera temporada de operaciones se exportaron 1979.889 cajas de banano tipo $\mathrm{XU}$, y desde entonces las cifras han ido aumentando vertiginosamente, manteniendo la alta calidad como constante en cada entrega. Palmar desde el inicio de sus actividades a mantenido un promedio de cajas de exportación con un volumen de 17 millones de cajas de banano tipo XU aproximadamente por año hasta la actualidad, estas cifras prueban que Palmar tiene un lugar de gran importante entre las productoras y exportadores bananeras ecuatorianas, y la han llevado a convertirse en una de las primeras Exportadoras de Banano del país.

Palabras clave: Sistemas, comercialización, Información, Productos, Mercado.

\footnotetext{
${ }^{1}$ Universidad Técnica de Ambato, Ambato, Ecuador, le.velastegui@uta.edu.ec ${ }^{2}$ Universidad Técnica De Cotopaxi Extensión "La Maná", Ecuador, erikherreravargas@hotmail.com 


\begin{abstract}
.
Bananas and plantains are perennial crops grow rapidly and can be harvested year-round. In the year 2000, an area of around 9 million hectares was cultivated. The average of world production in 1998-2000 was 92 million tons per year and in 2001 if was estimated at 99 million tons.

These figures are an approximation, since most of the world banana production, almost 85 percent, comes from relatively small plots and family gardens where there are no statistics.

Ecuador is a privileged country and internationally recognized as the leading occupies a very important place in the economic development of Ecuador, given the high annual banana export volumes it makes to markets whose standards are extremely demanding. Only during the first season of operations, 1979,889 boxes of type 22 XU bananas were exported, and since then the figures have been increasing rapidly, maintaining the high quality as constant in each delivery.

Palmar since the beginning of its activities has maintained an average of export boxes with a volume of 17 million boxes of type $22 \mathrm{UX}$ banana per year to date, these figures prove that Palmar has a place of great importance among producers and Ecuadorian banana exporters, and have led it to become one of the first banana exporters in the country.
\end{abstract}

Keywords: Production, Marketing, Banana \& Socio-economic aspect.

\title{
I. Introducción.
}

\section{Producción.}

Según los autores (Goncalves L. \& Kernaghan J. 2014., Morillo J. 2014., Palmar C. 2015., Pérez A. 2017., Poso T. 2014 \& Willemse W. 2018): Ecuador es un país privilegiado y reconocido internacionalmente como el primer exportador bananero en el mundo, destacándose por la calidad de su fruta. Corporación palmar ocupa un lugar muy importante en el desarrollo económico del Ecuador, dados los altos volúmenes anuales de exportación bananera que realiza a mercados cuyos estándares de calidad son sumamente exigentes. Ecuador es un país privilegiado y reconocido internacionalmente como el primer exportador bananero en el mundo, destacándose por su calidad de su fruta. Corporación Palmar ocupa un lugar muy importante en el desarrollo económico del Ecuador, datos los altos volúmenes anuales de exportación bananera que realiza a mercados cuyos estándares de calidad son sumamente exigentes el fruto de este cultivo se consume en estado maduro.

No obstante, se ha incrementado en los últimos años puesto se considera un alimento ideal para los niños, deportistas y personas de todas edad por su alto valor nutritivo. Este comportamiento se debe a la utilización de las variedades más productivas, mejoramiento de las condiciones físicas y químicas del suelo y a la presencia de condiciones climáticas favorables para el desarrollo del cultivo. 
Teniendo en cuenta todo esto existe un creciente interés por parte de consumidores, especialmente en los países del norte, por una producción responsable y formas más equitativas de Comercializando y distribuyendo el producto.

Se denomina producción a cualquier tipo de actividad destinada a la fabricación, elaboración u obtención de bienes y servicios. En tanto la producción es un proceso complejo, requiere de distintos factores que queden dividirse en tres grandes grupos, a saber: la tierra, el capital y el trabajo.

La tierra es aquel factor productivo que engloba a los recursos naturales; el trabajo es el esfuerzo humano destinado a la creación de beneficios; finalmente, el capital es un factor derivado de los notros dos, y representa al conjunto de bienes que además de poder ser consumido de modo combina los citados elementos para satisfacer las necesidades de la sociedad, a partir del reconocimiento de la demanda de bienes y servicios.

El banano es el principal rubro de exportación no petrolera del país. En el 2015 Ecuador exporto alrededor de 120 millones de cajas de la fruta. El cultivo de banano ha sufrido en el pasado una reducción en su producción debido a enfermedades como hongo de la sigatoka negra y todavía mantiene bajos rendimientos por hectárea, en comparación con otros países productores.

Grafico 1.- Producción.

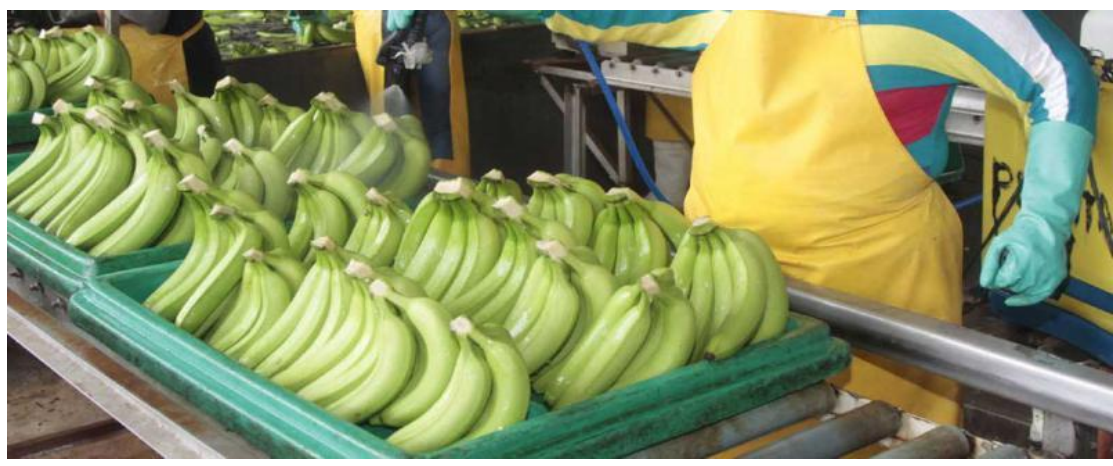

Fuente: www.revistaliders.ec.

\section{Comercialización.}

Según los autores (Canales L. 2015., Pascal L. 2017., Romel B. 2015., Urgiles H. 2016., Workman D. 2018): Exportaciones en 2015, las exportaciones mundiales de bananos, excluidos los plátanos, registraron su primer descanso desde 2010, tras haber alcanzado un nivel sin precedentes de 19,6 millones de toneladas en 2014. Aunque la demanda de importaciones siguió siendo fuerte en todas las regiones, los efectos adversos del fenómeno de El Niño, así como la propagación de la fusariosis del banano, afectaron negativamente a los rendimientos y produjeron una escasez de producción en varias de las principales productoras y exportadoras.

En el ámbito de la economía, exportación se define como él envió de un producto o servicio a un país extranjero con fines comerciales. Estos envíos se encuentran regulados por una serie de disposiciones legales y controles 
impositivos que actúan como marco contextuales de las relaciones comerciales entre países.

Servicios y productos apoyados en Vías terrenas, aéreas y más recientemente virtuales. La exportación puede efectivizarse a partir de las diferentes vías de transporte, porque si o si, la exportación el conjunto de bienes o servicios deben "viajar" hacia otro país, que se encuentra a varios kilómetros de distancia, y entonces será preciso que se los traslade por tierra, en camiones, automóviles, entre otros, por mar, o por avión.

En los últimos años gracias a las bondades que proporcionan ellas nuevas tecnologías, especiales Internet se ha vuelto algo común y frecuente que la gente exporte sus servicios laborables vinculados a labores vía web, y entonces, en este caso especial, lo que se exporta es un servicio abstracto.

La actividad opuesta a la de exportación es la de importación, que por el contrario supones ingreso, la instrucción de bienes o servicios de orig foráneo a una nación exportación balanza comercial.

Grafico 2.- Comercialización o Exportación.

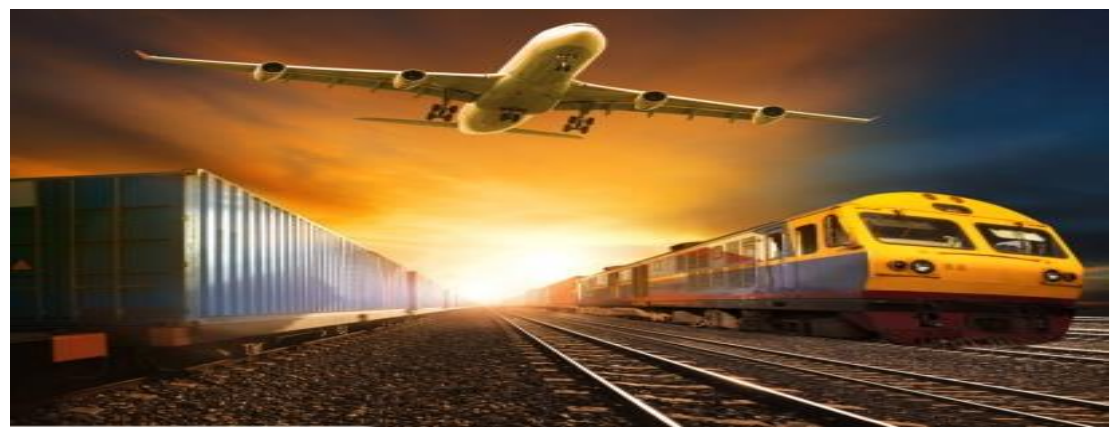

Fuente: www.definicionabc.com

En un mundo tan globalizado la balanza comercial es un elemento fundamental en el devenir de la economía de los países, ya que estos se encuentran inmersos en una red de intercambios de importaciones y exportaciones a nivel mundial. Lograr el equilibrio en esta balanza es uno de los objetivos de cualquier país que quiera mantener saneadas sus cuentas y no incurrir en un déficit excesivo. Explicando de manera intuitiva, para que un país mantenga una balanza comercial equilibrada no debe comprar más de lo que vende, o dicho de otro modo, las importaciones no deben superar a las exportaciones. 


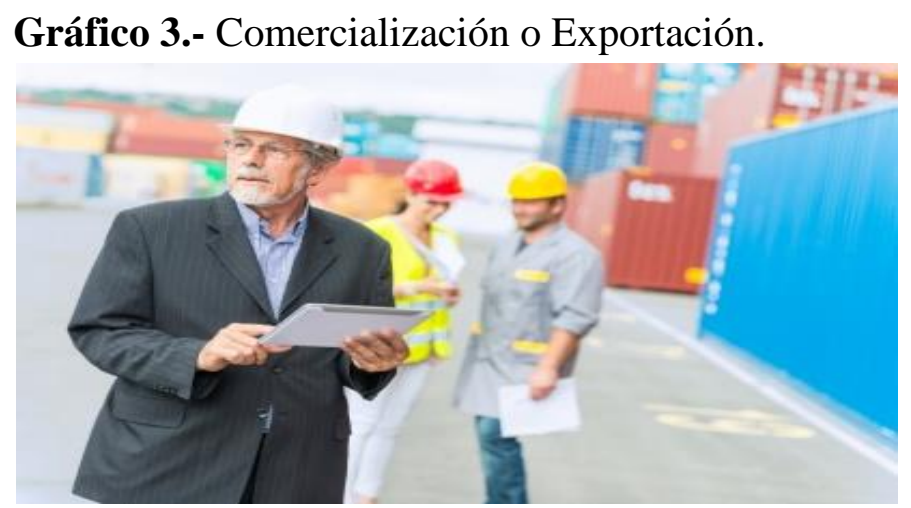

Fuente: www.definicionabc.com

Sin embargo, la variabilidad de las divisas es también un arma de doble filo, ya que puede afectar a alguna de las partes negativamente si la transacción se realiza en una moneda que experimenta un alza o baja muy acusada en un periodo de tiempo reducido. Hay que tener en cuenta que las exportaciones se cierran a un determinado precio y unas condiciones de pago concretas, que suelen incluir pagos aplazados a 90, 120, 0180 días, y que una variación sustancial del valor de la divisa entre uno y otro momento pueden terminar por generar importantes desequilibrios sobre precio acordado inicialmente.

\section{Banano.}

Según los autores (Aguilar R. 2015., Carrillo S. 2015., Gacthe R. 2015., Holanda S. 2016., Kukulies T. \& Veivers S. 2017., Quito C. 2015): El banano de postre es una de las primeras frutas que se comercializaron en todo el mundo: apareció en Europa y América del Norte a principios del siglo XIX procedente del Caribe.

El auge del comercio del banano se explica, por un lado, gracias a la mejora de la década logística, especialmente de las condiciones de trasporte (rapidez y refrigeración) y a la invención de la técnica de maduración de maduración tras el trasporte; y por otro, gracias a los números pioneros y aventureros que se lanzaron eb la producción, trasporte y comercialización del banano de postre entre los años 19870 y 1900 y que lo hicieron famoso en América del Norte.

El precio internacional de la fruta manifiesto similar tendencia al de la producción, debilitándose en mercados como Estados Unidos, Rusia, China, Japón. La variedad que tiene mayor acogida es la "Cavendish" que es la que se cosecha para las exportaciones. Cabe indicar que el mercado mundial de banano es muy amplio, ya que contamos con el $30 \%$ de la oferta mundial y la fruta representada el $10 \%$ de las exportaciones totales y es el segundo rubro de mayor importancia del país. Según los registros de producción el 95\% de la producción total es enviada al mercado extranjero, llegando así a más 43 países a nivel mundial. 
El principal beneficiario de este lamentable hecho que les sucedió a los países centroamericanos sin duda fue el Ecuador, decido a que desde ese entonces comenzó a aumentar la demanda de nuestro banano por parte de Europa y de los Estados Unidos.

Los plátanos de Canarias se diferencian por su sabor más dulce su aroma y sus inconfundibles motitas.

Esto es debido a que poseen un mayor índice de humedad, por lo que son mucho más jugosos que la banana, que es más seca y tienen una textura más harinosa por su composición en carbohidratos, azucares solubles y sacarosas.

Así describe la Asociación de Organizaciones de Productores de Plántanos de Canarias (Asprocan) las principales diferencias entre planta no y banana, dos frutas de la misma familia, pero con detalles que las distinguen, tanto en el peladar como en su nutrientes.

En España estamos más acostumbrados al consumo de plátanos, pero la banana también es una buena alternativa para ayudar al cuerpo a recuperarse tras un esfuerzo físico, como la práctica deportiva, aunque la cantidad de potasio (el mineral que más influye en esta recuperación) es algo superior en planta no.

La Banana contiene más almidón que el plátano pero también es mucho más rica en calcio imprescindible para fortalecer dientes, huesos y cabello.

En cuanto a las vitaminas, ambas frutas contiene vitaminas A, B2, B6, C y riboflavina, en cantidades superiores en el plátano respecto de la banana. Al ser un alimento menos dulce, la banana suele utilizarse en platos salados, como guisos, fritos u horno.

Disminuye la presión arterial gracias a que contiene el $23 \%$ desde la dosis diaria recomendada por ingesta de potasio.

Ayuda a que nuestros huesos absorban con mayor facilidad el calcio, ya que contiene altos niveles de fructoolgosacáridos.

Gráfico 4.- Banano.

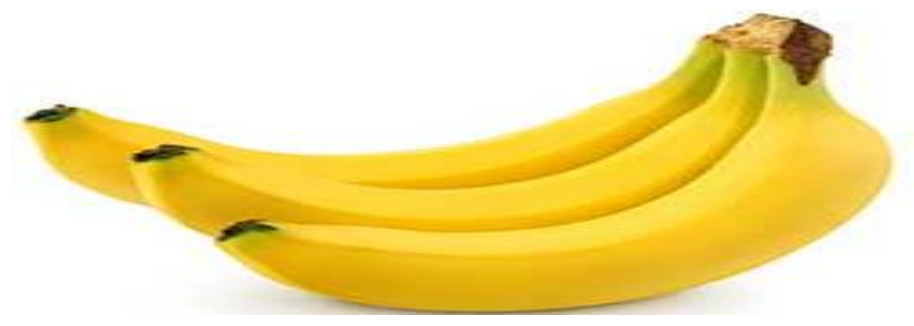

Fuente: collinsdictionary.com 


\section{Aspecto socio económico.}

Según los autores (Cooper A. 2015., Cuervo L. 2015., Elbehi A. 2015., Guzmán

K. \& Sánchez M. 2018., Ramírez E. 2015): Esta actividad se viene desarrollando en las ares de producción y explotación en los departamentos del Magdalena y Antioquia. Las áreas en producción de estas dos zonas comprenden 40.500 hectáreas de las cuales 30.000 hectáreas corresponden a la Zona de Urabá y 10.500 hectáreas en la Zona de Magdalena el sector bananero tiene una importancia vital para el Ecuador debido a sus dimensiones y al gran porcentaje de la población (10 por ciento) que obtiene sus medios de subsistencia económicos de dicho sector.

El sector emplea 21.00 trabajadores directos en Urabá y 4.000 en Magdalena; empleos de los que se benefician más de 1000.000 familias.

El acuerdo comercial con la Unión Europea, tiene una gran significado según los datos de la organización Mundial del Comercio (OMC), en relación con nuestro país Europa es el primer importador con cifras aproximadas de 5.883 millones de euro y las exportaciones están en 8.9292 millones de euros, las mujeres representan menos de una quinta parte de la fuerza laboral mundial en la industria de exportación de banano.

La investigación encontró la mayor participación de mujeres en la industria en el Caribe excluyendo a los dominicanos económicos del cultivo el rio es el principal fuente de agua de riego para la producción de banano.

El nivel o estatus socioeconómico es una medida total económica y sociológica combinada de la preparación laboral de una persona, de la posición económica y social individual o familiar en relación a otras personas, basada en sus ingresos, educación u empleo al analizar el nivel socioeconómico de una familia se analizan, os ingresos del hogar los niveles de orientación educación, ocupación, como también el ingreso combinado, comparado con el individual, también son analizados los atributos personarles de sus miembros.

En la actualidad nuestro país se encuentra pasando por una etapa muy importante modificara su estructura económica, todos estos cambios han venido siendo notorios desde la apertura económica de nuestros mercados, pero este tiempo no sirvió para que las empresas se fortalecieran, al contrario la globalización nos tomó poco preparados, con economía nacional.

La mayoría de los peruanos vivían de la exportación, trasformación y exportación de los recursos naturales, de la agricultura y de los servicios. La fuerte crisis económica de finales de los 80, agudizo más aun los problemas, hasta la aplicación de una drástica política de apertura neoliberal y corrección de las cuentas fiscales en los 90 , lo cual ha permitido el despeguen económico del país. 
Grafico 5.- Aspecto socio económico.

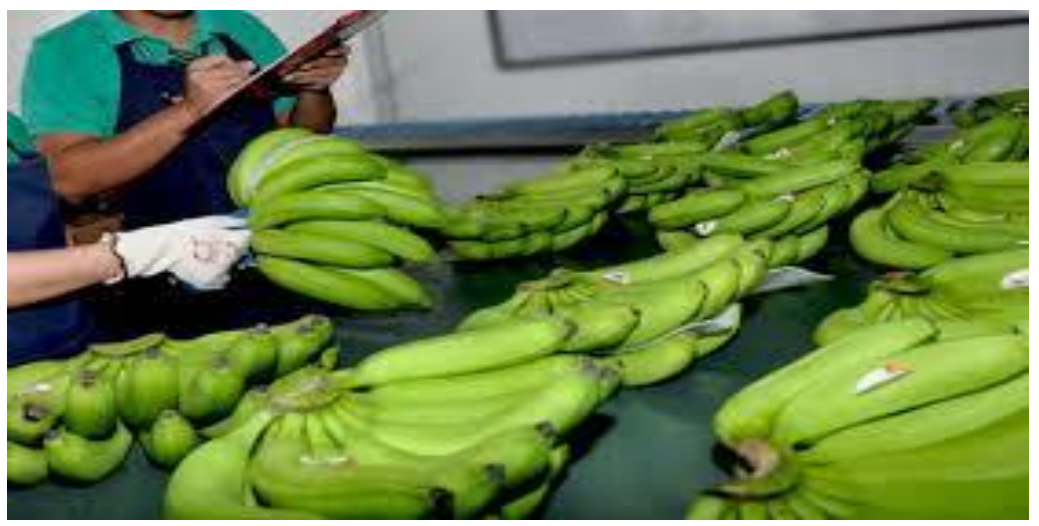

Fuente: www.google.com.

Datos estadísticos de la comercialización del banano en el ámbito mundial:

Grafico 6.- Datos estadísticos Mundial.

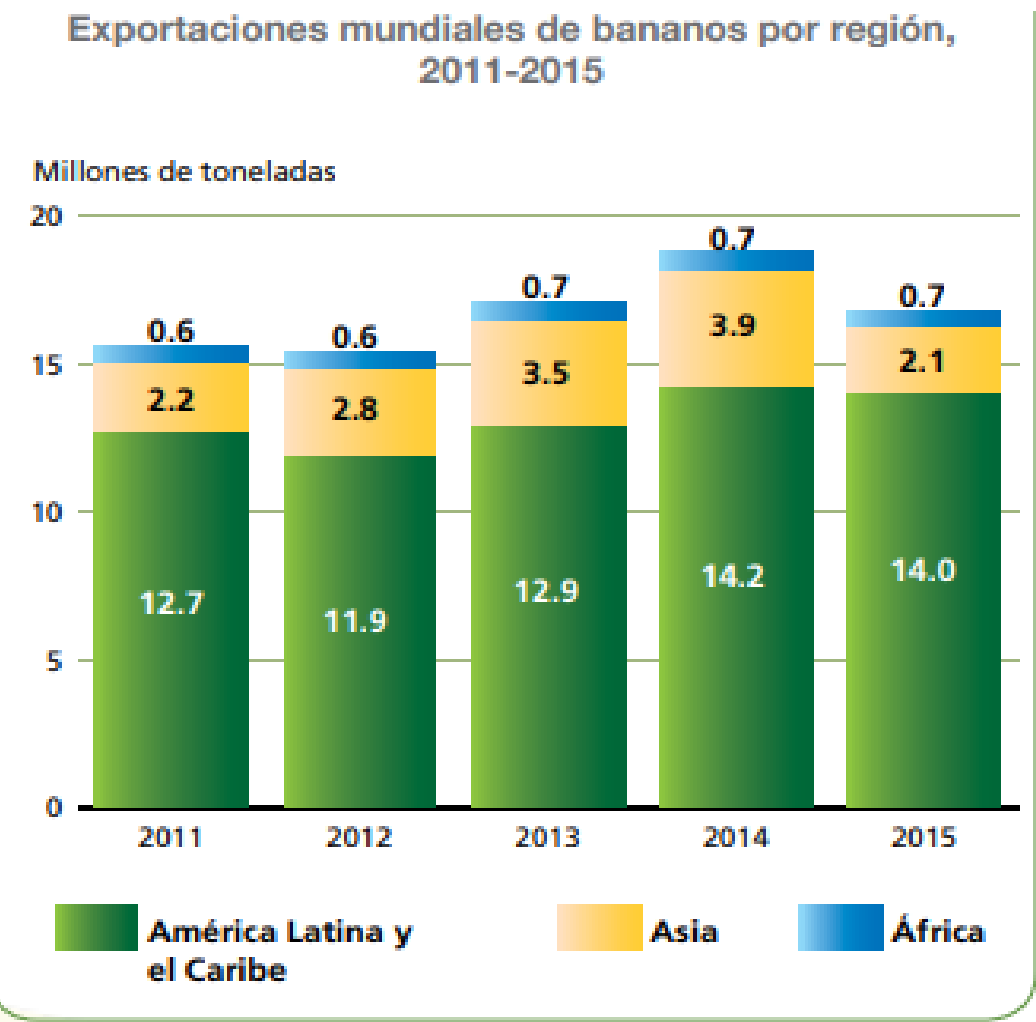

Fuente: http://www.fao.org/3/a-i7410s.pdf 
Las condiciones climáticas adversas y la fusariois del banano afectaron gravemente a la producción en Filipinas. La India, con mucho el mayor productor mundial de bananos, aumento su volumen de exportación en un $47 \%$ debido a una nueva expansión de la superficie cosechada para las variables comercializadas. Aunque la producción de banano en la India se destina principalmente al mercado interno, una parte creciente de la misma se exporta a los países del Golfo, Malasia y Nepal. La escasez de suministros en Filipinas, el principal exportador competidor, implico que los envíos de la India pudieran beneficiarse de la alta demanda de los países del Golfo y el sudeste asiático.

Otro factor de apoyo fue el bajo precio de los bananos de la India, que según se informa se vendieron con un descuento del $50 \%$ respecto de los bananos del Ecuador y Filipinas en la subasta de Dubái las exportaciones africanas, que representaron en 3,9\% de los envíos mundiales de bananos, se redujeron un 12\% en 2015, situándose en 604000 toneladas, debido principalmente a la grave escasez producción en Ghana. Cote d'lvoire el mayor exportador de la región, envió 305000 toneladas de banano, un $9 \%$ menos que el año anterior, después de que las inundaciones destruyeran gran parte de la cosecha en junio de 2014. Las exportaciones de Camerún, a pesar de los costos a los que hizo frente para luchar contra la sigatoka negra. Cote d'lvoire y Camerún exportan del $80 \%$ de la producción nacional, siento Europa el principal destino de sus exportaciones.

\section{Datos estadísticos de la comercialización del banano en Latino América:}

Grafico 7.- Datos estadísticos Latino América.

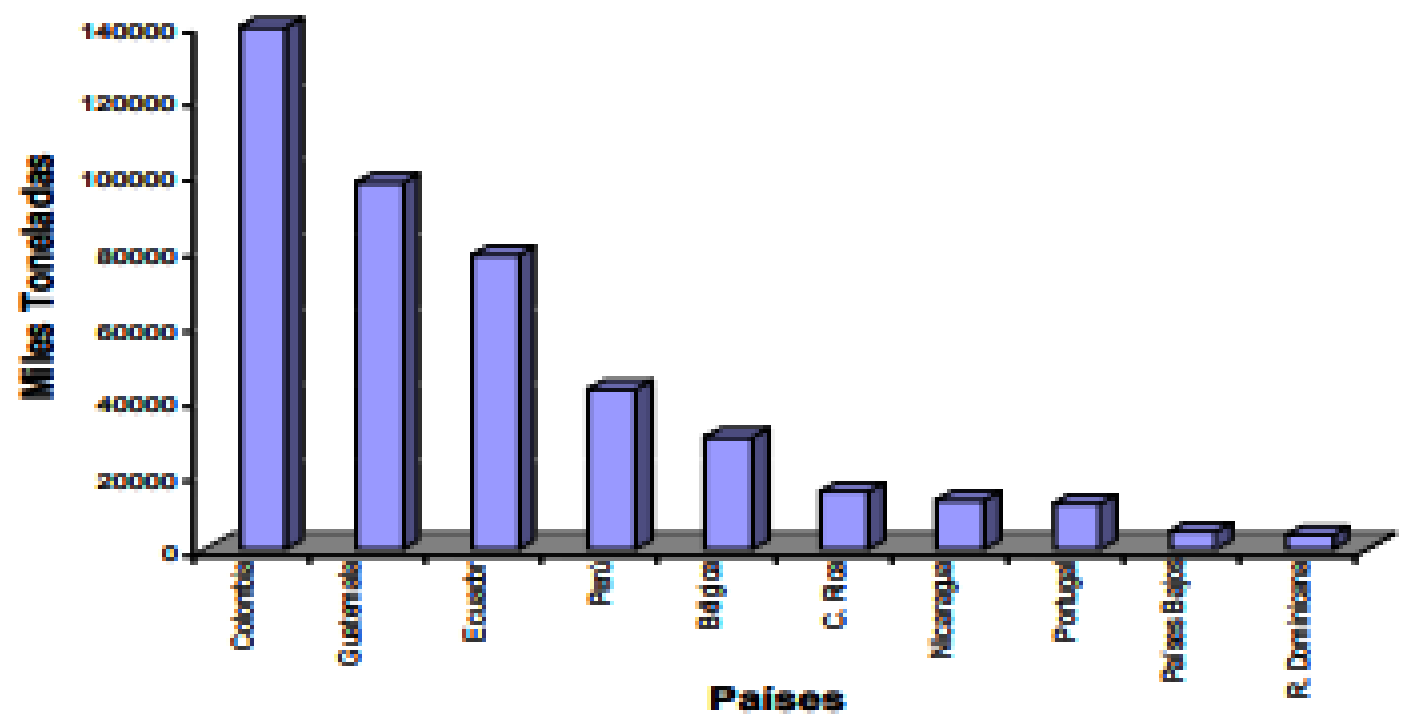

Fuente: file:///C:/Users/DELL/Downloads/4.c.11.pdf

Los países exportadores de plátano, por lo general, son los mismos países exportadores de banano pero a diferencia del banano, el plátano no ha tenido consumo masivo en 
ISSN 2602-8506

Vol. 1 / No.2/ pág. 39-52. Abril-Junio/2017

www.visionariodigital.org

Estados Unidos y Europa, haciendo que estas exportaciones estén destinadas especialmente al mercado latino y afroamericano. Las exportaciones del continente americano tienen como líder a Colombia, seguido por Guatemala, Ecuador y Perú; mientras que en el continente europeo las exportaciones están lideradas por Bélgica (posición 5), Portugal (posición 8), Países Bajo(posición 10), Italia (posición 13), España (posición 15), Francia (posición 16), otras naciones como Alemania, Irlanda y Reino Unido contribuyen con un menor porcentaje.

Debe indicarse la importancia de la participación de algunos países en este renglón, ya que contribuyen de manera determinante dentro del proceso de exportación del cultivo, y distribución de su fruta, tal es el caso de Bélgica que no es un país productor de plano pero aparece con importancia marcada en la distribución. Es la producción proveniente de Colombia y de sus ex colonias destaca que la mayor parte de los países exportadores del mundo se encuentran en América Latina.

Datos estadísticos de la comercialización del banano en Ecuador:

Tabla 1.- Datos estadísticos en Ecuador. 


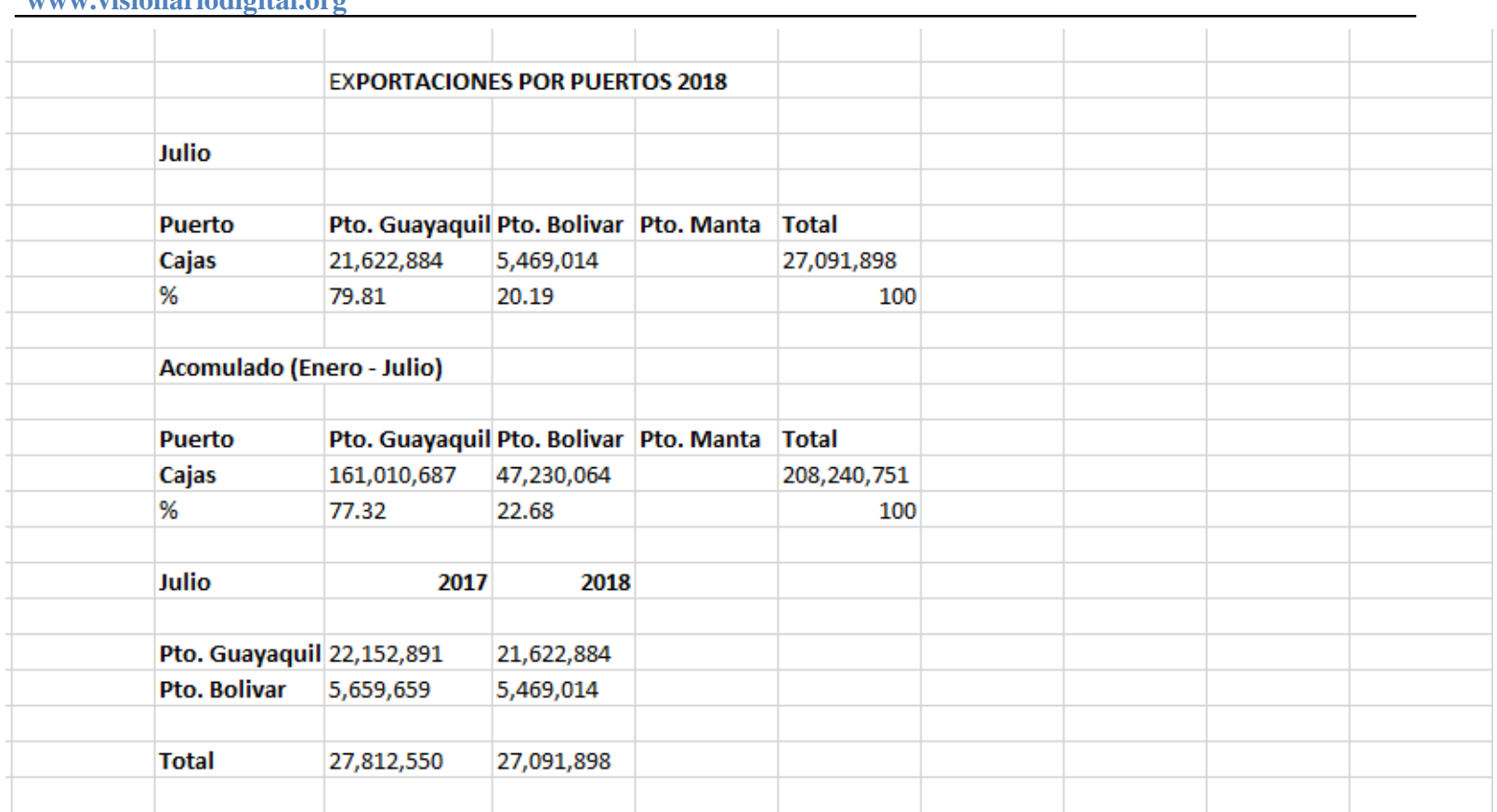

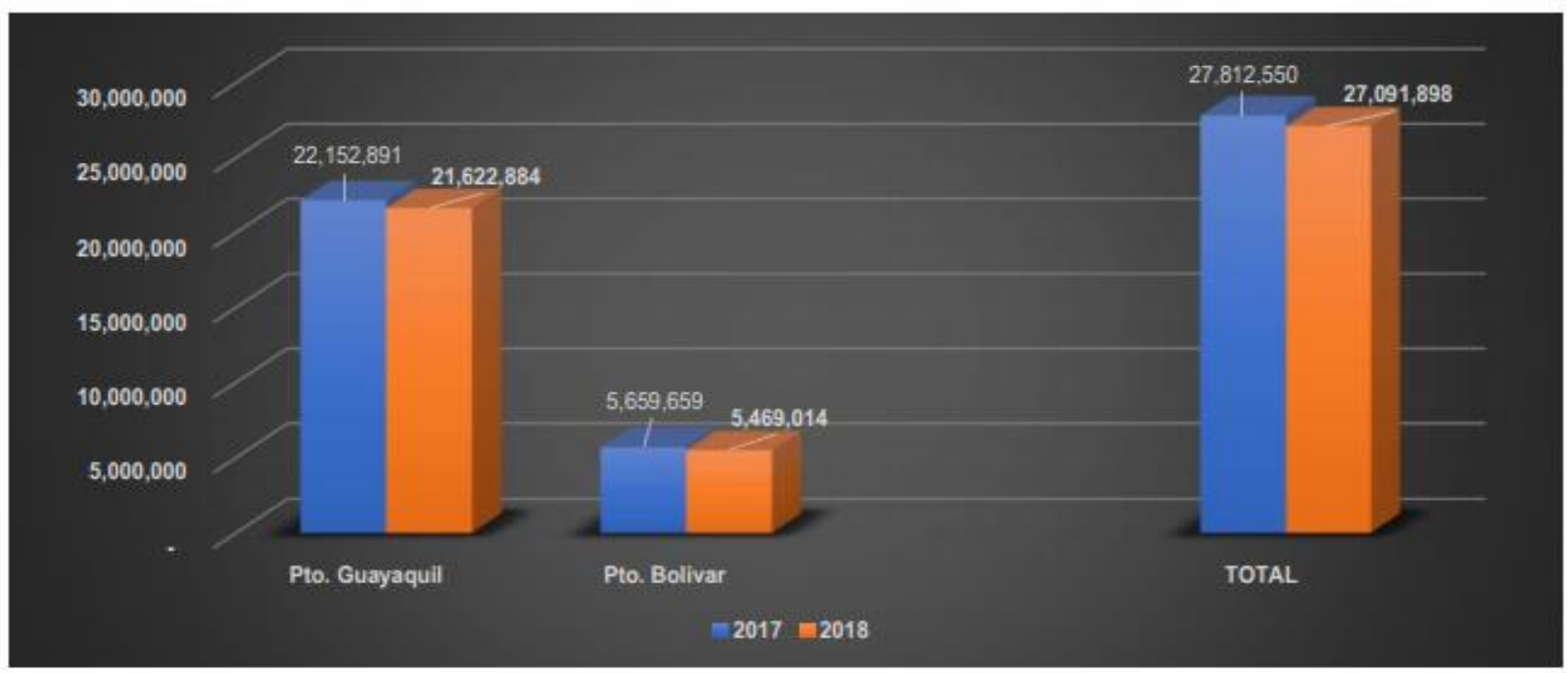

Fuente:www.aebe.com.ec/wpcontent/uploads/2018/10/AE_EstadisticasP ublicas_Jun18.pdf

Los cinco principales destinos de los envíos de la fruta durante este año han sido Unión Europea, Rusia, Estados Unidos, Argentina y Turquía, Las exportaciones de banano ecuatoriano se incrementaron en un 12\% entre enero y octubre del 2017 y el mismo periodo de 2016. Según datos del Banco Central del Ecuador (BCE), en dicho lapso de 2017, se han vendido un total de 5.333 toneladas de la fruta por $\$ 2.490$ millones (FOB). El principal destino del producto ecuatoriano fue la Unión Europea, con una participación del 35\%; seguidos de Rusia con el 24\$\%; y en tercer lugar, Estados Unidos con el 18\%. Eduardo Ledesma, presidente de la Asociación de Exportadores de Banano del Ecuador (AEBE) estima que este año se cerrara con la exportación de unas 325 millones de cajas. Según la Corporación de Regional de Bananeros Ecuatorianos (Agroban), el año pasado se vendieron entre 300 y 315 millones. 
A pesar del crecimiento del sector, Ledesma aseguro que el comercio de la fruta tiene múltiples problemas como los reglamentos vigentes y tramites que frenan el desarrollo de la actividad. La competitividad del producto también se ha visto frenada en mercados como el estadunidense. Ledesma explico que está perdida se dio porque los costos del banano mexicano y guatemalteco son inferiores a los del ecuatoriano, son embargo espera recuperar este nicho.

El mayor productor mundial de bananos aumento su volumen de exportación en un 47\% debido a una nueva expansión de la superficie cosechada para las variedades comercializadas, Camerún es el segundo exportador africano aumento un 6,5\% a 283000 toneladas. Las exportaciones del continente americano tienen como líder a Colombia, segundo por Guatemala, Ecuador y Perú, en el caso de Bélgica que no es un país productor de plátano pero aparece como importancia marcada en la distribución, la mayor parte de los países exportadores del mundo se encuentra en América Latina.

Los cinco principales destinos de los envíos de la fruta son la Unión Europea, Rusia, Estados Unidos, Argentina y Turquía, el principal destino ecuatoriano fue la Unión Europea con una participación del 35\%; segundo de Rusia con el 24\% y el tercer lugar Estados Unidos con el 18\%, el presidente de la Asociación de Exportadores de banano del Ecuador estima que este año se cerrara con la exportación de unas 325 millones de cajas.

\section{Conclusiones.}

- Crear una comisión de negociadores por parte del gobierno Ecuatoriano y la empresa privada del sector bananero, con el propósito de lograr un tratado bilateral de comercio con Brasil que permita obtener tarifas preferenciales para la exportación de banano y otros productos perecibles.

- El mercado Europeo es muy exigente en cuanto a los estándares de la calidad de la fruta por lo que el sector bananero implemento un sistema d control para realizar el proceso de cultivación de la forma adecuada, cumplimiento de estándares de calidad y control de plagas.

- Establecer un plan de contingencia para reducir los daños producidos por las plagas y enfermedades mediante la implementación de un sistema de control para el proceso de cultivación del banano cumplimiento con los estándares de calidad.

- Brindar capacitaciones a los productores de banano para que sepan de qué manera llegar a tener un mayor beneficio de sus cultivos, para de esta manera incrementar las fuentes de empleo. 
- Otorgar por parte de gobierno mayor financiamiento a los productor a los productores del sector bananero para que puedan diversificar su oferta y pasar de ser exportadoras de banano como materia prima a ser exportadoras de productoras de productos elaborados a base de banano, sí se dinamizaría el sector exportador e industrial del país.

\section{Referencias.}

Aguilar .r. (2015). "la producción y exportación del banano y su incidencia en la economía ecuatoriana. Guayaquil - ecuador.: facultad de ciencias económicas.

Canales .1. (2015). Plan de exportacion de pure de banano para la asociacion de pequeños productores bananeros de el guabo. El oro: utmch.

Carrillo .s. (2015). Banana. New york y ginebra: fundo de la unctad.com.

Gaethe .r. (2015). Boletin situacional banano. Ministerio de agricultura y ganaderia.

Gonçalves .1 \& kernaghan .j. (2014). Banana production methods. Costa rica: comparative study.

Holanda .s. (2016). Banano. Quito: national institute for agricultural research.

Kukulies .t \& veivers .s. (2017). Banana. Australia: on-farm biosecurity.

Morillo .j. (2014). Produccion de banano. Quito-ecuador: agricultura s.a.

Palmar .c. (2015). Producción de banano. Quito: alfa digital s.a.

Pascal. Liu. (2017). Situacion del mercado del banano. Roma: fao.com.

Perez .a. (2017). El poductor. Guayaquil: el productor.

Poso .t. (2014). Produccion de banano. Quito-ecuador: inamhi.

Quito .e. (2015). Plan de exportacion de pure de banano para la asociacion de. Machalael oro: utmch.

Rome .a. (2017). Banana. Manabi: agriculture organization of the united nations.

Rómmel .b. (2015). Impacto potencial en las exportaciones de banano. Quito: facultad de economía.

Urgiles .h. (2016). Proyecto de factibilidad para la exportación de banano deshidratado al mercado. Quito: facultad de ciencias económicas y negocios.

Willemse .w . (2018). Production banana. Canada: arc-institute for tropical and subtropical.

Workman .d. (2018). Exported bananas. Canada: bananas exports by country.

mekonnen .a. (2016). Banana as a cash crop and its food security and socioeconomic contribution: the case of southern ethiopia, arba minch. Ethiopia: university of leicester, leicester, uk.

Cooper .a. (2015). Bananalink. Canada: world banana forum.

Cuervo .1. (2015). Aspecto socio economico. Colombia: servicio nacional de aprendizaje.

Elbehri .a . (2015). Cambio climatico y sostenibilidad del banano en el ecuador. Roma: organización de las naciones unidas para la alimentación.

Guzmán .k \& maría sánchez .a. (2018). Condición actual del proceso exportador de banano colombiano hacia la unión europea. Colombia: universidad del rosario.

Ramírez .e. (2015). Informe socioeconómico. Uraba: cámara de comercio de urabá. 


\section{Para citar el artículo indexado.}

Velasteguí E \& Herrera E. (2017). Sistema de información de mercado para el sector de agro alimentos de los mercados de la provincia de Tungurahua. Revista electrónica Visionario Digital 1(1), 39-52. Recuperado desde: http://cienciadigital.org/revistacienciadigital2/index.php/VisionarioDigital/article/view/ $\underline{241 / 537}$

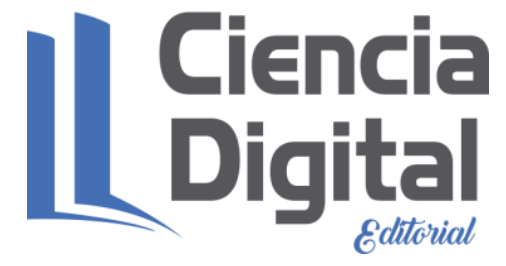

El artículo que se publica es de exclusiva responsabilidad de los autores y no necesariamente reflejan el pensamiento de la Revista Ciencia Digital.

El articulo queda en propiedad de la revista y, por tanto, su publicación parcial y/o total en otro medio tiene que ser autorizado por el director de la Revista Ciencia Digital.
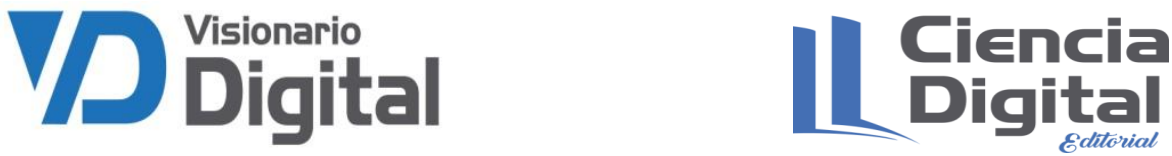